\title{
Association between ibuprofen exposure and severe COVID-19 infection: a nationwide register- based study
}

Kristian Kragholm ( $\nabla$ kdks@rn.dk)

Aalborg University Hospital

Thomas A. Gerds

Copenhagen University

Emil Fosbøl

Copenhagen University Hospital

Mikkel Porsborg Andersen

Nordsjaellands Hospital

Matthew Phelps

Danish Heart Association

Jawad H. Butt

Copenhagen University Hospital

\section{Lauge Østergaard}

Copenhagen University Hospital

\section{Gunnar Gislason}

Gentofte and Herlev University Hospital

Morten Schou

Gentofte and Herlev University Hospital

Lars Køber

Copenhagen University Hospital

Christian Torp-Pedersen

Nordsjaellands Hospital

\section{Research Article}

Keywords: NSAID, ibuprofen, COVID-19, disease severity, mortality.

Posted Date: May 1st, 2020

DOI: https://doi.org/10.21203/rs.3.rs-26247/v1 
License: (c) (i) This work is licensed under a Creative Commons Attribution 4.0 International License. Read Full License

Version of Record: A version of this preprint was published at Clinical and Translational Science on May 1st, 2020. See the published version at https://doi.org/10.1111/cts.12904. 


\section{Abstract}

Background: Initially, the World Health Organization (WHO) and national health boards issued a warning against NSAID use in corona virus disease 2019 (COVID-19) patients and recommended that paracetamol or acetaminophen instead should be administered. However, later and current WHO and European Medicine Agency recommendations do not call for avoidance of ibuprofen in COVID-19 patients. Given the current uncertainty of drug safety for NSAID use in patients with COVID-19, we performed a nationwide register-based study on the association of recent ibuprofen exposure and COVID19 severity.

Methods: Using national administrative databases, we identified COVID-19 patients in Denmark between end of February 2020 and April 2, 2020. Patients <30 years of age and heart failure, for whom ibuprofen is not recommended, were excluded. Ibuprofen exposure was defined using the Danish National Prescription Registry, where we had information until January 31, 2020. Endpoint was a composite of severe COVID-19 diagnosis with acute respiratory syndrome, intensive care unit admission or death.

Results: Among 1,872 patients, 46 (2.5\%) were exposed to ibuprofen prior to COVID-19 infection. Patients with recent ibuprofen exposure tended to be older and more likely to have hypertension, diabetes, myocardial infarction, chronic obstructive pulmonary disease, and cancer, though all insignificant $(P>0.05)$. When adjusting for these covariates, odds ratio was 1.57 [95\% $\mathrm{Cl} 0.72-3.38$ ], with 12 ibuprofenexposed patients meeting the endpoint (26.1\% [95\% Cl 13.4-38.8\%]) versus 272 unexposed patients (14.9\% [95\% Cl 13.4-16.4\%]), P=0.15.

Conclusion: The association between ibuprofen and severe COVID-19 was insignificant, although with a trend towards increased disease severity risk.

\section{Background}

The corona virus disease 2019 (COVID-19) pandemic caused by severe acute respiratory syndrome corona virus 2 (SARS-CoV-2) has led to substantial changes in healthcare utilization, morbidity, intensive care resources, and mortality (1-7). The entry point for this novel coronavirus for its further pathogenesis in humans is thought to be through an angiotensin converting enzyme 2 (ACE2) found in cell surfaces in the lungs, arteries, heart, kidney and intestines (8). It has been hypothesized that a number of drugs including non-steroidal anti-inflammatory drugs (NSAIDs) might facilitate and aggravate infection with COVID-19 (9-10). The mechanisms through which ibuprofen and other NSAIDs may accelerate COVID-19 infection and lead to further deterioration include upregulation of the ACE2 enzyme expression as well as these drugs may delay diagnostics by masking inflammation and fever (9-10). At first, the World Health Organization (WHO) and national health boards issued a warning against the use of NSAIDs in COVID-19 patients and that paracetamol or acetaminophen instead should be administered. However, later and current WHO and European Medicine Agency recommendations do not call for avoidance of ibuprofen to treat COVID-19 symptoms, based on the lack of evidence to support the initially recommended warning 
(10). Therefore, given the current uncertainty of drug safety for NSAID use in patients with COVID-19, we performed a nationwide register-based study on the association of recent ibuprofen exposure in COVID19 positive patients and severe infection defined as a diagnosis of COVID-19 infection with severe acute respiratory syndrome and/or intensive care unit utilization and/or fatal COVID-19 infection. Since very few patients were prescribed other NSAIDs, we only studied the association between ibuprofen exposure and outcome.

\section{Methods}

\section{Study setting and population}

In Denmark, all citizens hold a unique civil registration number used in all healthcare and social contacts and recorded in national administrative databases. From the Danish National Patient Registry, we identified COVID-19 positive patients between end of February 2020 and April 2, 2020, through use of ICD10 diagnosis codes DB342, DB342A and DB972 for unspecific COVID-19 infection and DB972A for COVID-19 infection with severe acute respiratory syndrome. Patients below 30 years of age as well as patients with heart failure, for whom ibuprofen is not recommended, were excluded.

\section{Exposure}

From the Danish National Prescription Registry, data on prescription medication was available until February 1, 2020; thus, we assessed ibuprofen exposure in a one-month span from January 1 to January $31,2020$.

\section{Covariates}

We included information on patient age and sex from the Danish Civil Registration System. We assessed the following comorbidities: Hypertension, diabetes, prior myocardial infarction, chronic obstructive pulmonary disease, hypertension, any malignancy, and rheumatic disease. Hypertension was defined as redemption of at least two antihypertensive drugs in two consecutive 100-day periods prior to the COVID19 diagnosis, as done previously (11). All other comorbid conditions were identified from the Danish National Patient Registry up to five years prior to COVID-19 diagnosis.

\section{Endpoint}

The study endpoint was a composite of severe COVID-19 diagnosis (ICD-10: DB972A), admission to an intensive care unit (ICU), or death. The Danish National Patient Registry was used to identify the first two 
components of the composite endpoint, whereas vital status was identified from the Danish Civil Registration System.

\section{Statistics}

Continuous variables are reported using median and 25-75 percentiles. Categorical variables are reported using counts and percentages. Propensity for ibuprofen treatment was evaluated using logistic regression. Furthermore, we used unadjusted, age- and sex-adjusted, and fully adjusted logistic regression to associate the risk of severe COVID-19 infection, death or intensive care unit admission with ibuprofen exposure. Fully adjusted models included the following covariates: age, sex, diabetes, prior myocardial infarction, chronic obstructive pulmonary disease, hypertension, any malignancy, and rheumatic disease. Age was divided into the following categories: $30-50,>50-60,>60-70$, and $>70$ years. Reported are odds ratios with $95 \%$ confidence intervals $(\mathrm{Cl})$. Data management and analyses were performed using $\mathrm{R}$, version 3.6.1 (12).

\section{Ethics}

Register-based studies do not require ethical committee approval or patient consent according to Danish legislation. In accordance with the General Data Protection Regulation (GDPR), the data responsible institute in the Capital Region of Denmark has approved the use of the data sources for research purposes (approval number P-2019-191). Data are accessed on secure servers under Statistics Denmark and cannot be shared according to Danish legislation.

\section{Results}

\section{Patients and characteristics}

Of a total of 2,220 patients with COVID-19 infection between end of February and April 2, 2020, 1,872 patients without heart failure and age $\geq 30$ years formed the study population (Figure 1). A total of 46 patients (2.5\%) were exposed to ibuprofen prior to COVID-19 infection. Patients with recent ibuprofen exposure tended to be older and more likely to have hypertension, though insignificant, as well as the prevalence of diabetes, myocardial infarction, chronic obstructive pulmonary disease, and cancer were numerically higher for patients with ibuprofen exposure (Table 1). 


\section{Propensity of ibuprofen treatment}

Propensity of ibuprofen treatment for variables listed in Table 1 are shown in Table 2. None of these factors were significantly associated with a propensity for ibuprofen use, although there was a trend towards an increased use for hypertension patients (Table 2).

\section{Outcome}

A total of $315(16.8 \%)$ patients met the composite endpoint of severe COVID-19 infection (diagnosis with severe acute respiratory syndrome, ICU admission, or death). For ibuprofen exposed patients, 12 patients met the endpoint corresponding to $26.1 \%$ [ $95 \% \mathrm{Cl} 13.4-38.8 \%$ ] versus 272 patients corresponding to $14.9 \%$ [95\% $\mathrm{Cl} 13.4-16.4 \%]$ among those not exposed to ibuprofen $(P=0.15)$. The association between ibuprofen and the severe COVID-19 composite endpoint was insignificant, although with a trend towards an increased risk of the composite endpoint, with a fully adjusted OR of 1.57 [95\% $\mathrm{Cl} 0.72-3.38$ ], see Table 3.

\section{Discussion}

In this nationwide study on the association between ibuprofen exposure and severe COVID-19 infection, defined as a COVID-19 diagnosis with severe acute respiratory syndrome, intensive care unit admission, or fatal trajectory, we found no significant association between recent ibuprofen drug exposure and the composite endpoint.

Respiratory, septic and cardiovascular complications and prolonged recovery have been linked to NSAID use in COVID-19 infectious disease $(9,13)$. Initially, the WHO and several national health boards and experts advocated against the use of NSAID in COVID-19 infected patients and recommended that paracetamol or acetaminophen instead should be administered (13). Later, the WHO changed track and now currently do not call for avoidance of ibuprofen to treat COVID-19 symptoms. The American Food and Drug Administration (FDA) has stated their awareness of reports of potential worsening of COVID-19 infection on the basis of NSAID use, but the agency also stresses that there is currently insufficient evidence to support that NSAID should worsen the clinical course of COVID-19 infection. Our results do not provide sufficient evidence to change the current recommendations. However, although the relation between recent ibuprofen exposure and severe COVID-19 infection was insignificant in our study, the odds ratio was 1.57 with a $95 \%$ confidence interval between $0.72-3.38$. As such, we cannot rule out an insignificant result due to a type II error (insufficient power).

On the other hand, a significant relation between ibuprofen and severe COVID-19 infection could be a result of confounding by indication, given the observational nature of our study. In our study, ibuprofen users were defined on the basis of prescription fills and since ibuprofen also can be purchased as an over-the-counter (OTC) drug in Denmark, there are number of limitations related to ibuprofen exposure in 
our study. Apart from potential misclassification of ibuprofen exposure as unexposed, when in fact patients were taking OTC ibuprofen, patients on prescription ibuprofen are likely to be different than patients on OTC ibuprofen or patients not treated with ibuprofen with regard to underlying conditions both related and unrelated to the drug indication for ibuprofen. Therefore, although insignificant, the trend we see towards an increased risk of severe COVID-19 infection in our data may be related to conditions associated with ibuprofen use. However, in our analysis of propensity of ibuprofen treatment, no factors shown in Table 1 were significantly related to ibuprofen use, although a trend towards an increased use in patients with hypertension was seen.

Another potential source of misclassification of the ibuprofen exposure in our study is related to the time period during which we were able to assign patients as exposed versus unexposed to ibuprofen. We were only able to assess NSAID drug redemptions until the end of January, 2020, and the first positive COVID19 case in Denmark was in the end of February, 2020. Although we can argue that ibuprofen prescription was not made on the basis to treat COVID-19 infection, we cannot exclude that there were patients exposed to ibuprofen in February and onwards, but not in January. Since those patients then would be incorrectly registered as unexposed, results would be directed towards no association between ibuprofen and COVID-19 severity, and we cannot rule out that this misclassification has influenced the insignificant results seen in our study.

In addition to the limitations mentioned above, follow-up data on outcome was limited to April 2, 2020, which may limit the registration of severe COVID-19 infection for patients diagnosed with COVID-19 in the end of the study period. Given the observational design, results are associations, and no conclusions with regard to causality can be made. Although we adjusted for several confounding factors, we cannot rule out residual or unmeasured confounding. Despite the lack of laboratory data to confirm that each case had a positive swab test, coding of both tested persons with tentative diagnosis codes and eventually those positive swabs with definite diagnosis codes have been and are systematically performed in Denmark. Although most COVID-19 infections in Denmark are located to the capital and more densely populated areas, the use of nationwide data with consecutively included patients with positive COVID-19 infections minimizes selection bias.

\section{Conclusions}

In conclusion, we did not find ibuprofen drug exposure to be associated with severe and fatal trajectory in COVID-19 infection, but further investigations are needed to confirm our preliminary results.

\section{Declarations}

\section{Conflicts of interest}

None of the authors have any conflicts of interest to report. 


\section{Funding}

Departmental funding only.

\section{Authors' contributions}

KK, CTP, ELF, GG, MS and LK designed the study. KK, TAG, MPA, MP, CTP, GG, LØ and JHB aided in data acquisition and data management. KK, CTP and TAG analyzed the data. All authors read and approved the final manuscript.

\section{References}

1. Zhu N, Zhang D, Wang W, Li X, Yang B, Song J, Zhao X, Huang B, Shi W, Lu R, Niu P, Zhan F, Ma X, Wang D, Xu W, Wu G, Gao GF, Tan W; China Novel Coronavirus Investigating and Research Team. A Novel Coronavirus from Patients with Pneumonia in China, 2019. N Engl J Med. 2020 Feb 20;382(8):727-733. doi: 10.1056/NEJMoa2001017. Epub 2020 Jan 24.

2. Emanuel EJ, Persad G, Upshur R, Thome B, Parker M, Glickman A, Zhang C, Boyle C, Smith M, Phillips JP. Fair Allocation of Scarce Medical Resources in the Time of Covid-19. N Engl J Med. 2020 Mar 23. doi: 10.1056/NEJMsb2005114.

3. Grasselli G, Pesenti A, Cecconi M. Critical Care Utilization for the COVID-19 Outbreak in Lombardy, Italy: Early Experience and Forecast During an Emergency Response. JAMA. 2020 Mar 13. doi: 10.1001/jama.2020.4031. [Epub ahead of print] No abstract available.

4. Kinross P, Suetens C, Gomes Dias J, Alexakis L, Wijermans A, Colzani E, Monnet DL, European Centre For Disease Prevention And Control Ecdc Public Health Emergency Team. Rapidly increasing cumulative incidence of coronavirus disease (COVID-19) in the European Union/European Economic Area and the United Kingdom, 1 January to 15 March 2020. Euro Surveill. 2020 Mar;25(11). doi: 10.2807/1560-7917.ES.2020.25.11.2000285. Epub 2020 Mar 16.

5. Boccia S, Ricciardi W, loannidis JPA. What Other Countries Can Learn From Italy During the COVID-19 Pandemic. JAMA Intern Med. 2020 Apr 7. doi: 10.1001/jamainternmed.2020.1447.

6. Rubino S, Kelvin N, Bermejo-Martin JF, Kelvin D. As COVID-19 cases, deaths and fatality rates surge in Italy, underlying causes require investigation. J Infect Dev Ctries. 2020 Mar 31;14(3):265-267. doi: 10.3855/jidc. 12734 .

7. Grasselli G, Zangrillo A, Zanella A, Antonelli M, Cabrini L, Castelli A, Cereda D, Coluccello A, Foti G, Fumagalli R, lotti G, Latronico N, Lorini L, Merler S, Natalini G, Piatti A, Ranieri MV, Scandroglio AM, Storti E, Cecconi M, Pesenti A; COVID-19 Lombardy ICU Network. Baseline Characteristics and Outcomes of 1591 Patients Infected With SARS-CoV-2 Admitted to ICUs of the Lombardy Region, Italy. JAMA. 2020 Apr 6. doi: 10.1001/jama.2020.5394. 
8. Kuba K, Imai Y, Rao S, Gao H, Guo F, Guan B, Huan Y, Yang P, Zhang Y, Deng W, Bao L, Zhang B, Liu G, Wang Z, Chappell M, Liu Y, Zheng D, Leibbrandt A, Wada T, Slutsky AS, Liu D, Qin C, Jiang C, Penninger JM. A crucial role of angiotensin converting enzyme 2 (ACE2) in SARS coronavirusinduced lung injury. Nat Med. 2005 Aug;11(8):875-9. Epub 2005 Jul 10.

9. Fang L, Karakiulakis G, Roth M. Are patients with hypertension and diabetes mellitus at increased risk for COVID-19 infection? Lancet Respir Med. 2020 Apr;8(4):e21. doi: 10.1016/S22132600(20)30116-8. Epub 2020 Mar 11.

10. Russell B, Moss C, Rigg A, Van Hemelrijck M. COVID-19 and treatment with NSAIDs and corticosteroids: should we be limiting their use in the clinical setting? Ecancermedicalscience. 2020 Mar 30;14:1023. doi: 10.3332/ecancer.2020.1023. eCollection 2020.

11. Krogager ML, Torp-Pedersen C, Mortensen RN, Køber L, Gislason G, Søgaard P, Aasbjerg K. Short-term mortality risk of serum potassium levels in hypertension: a retrospective analysis of nationwide registry data. Eur Heart J. 2017 Jan 7;38(2):104-112. doi: 10.1093/eurheartj/ehw129.

12. R Core Team (2017). R: A language and environment for statistical computing. R Foundation for Statistical Computing, Vienna, Austria. URL https://www.R-project.org/.

13. Day M. Covid-19: ibuprofen should not be used for managing symptoms, say doctors and scientists. BMJ. 2020 Mar 17;368:m1086. doi: 10.1136/bmj.m1086.

\section{Tables}

Table 1. Characteristics of COVID-19 patients exposed and unexposed to ibuprofen. 


\begin{tabular}{|c|c|c|c|}
\hline Variable & Ibuprofen $(n=46)$ & No ibuprofen $(n=1,826)$ & P-value \\
\hline Age, median [25\%-75\%] & 60 [47-69] & $57[46-72]$ & 0.97 \\
\hline Age $30-50, \mathrm{n}(\%)$ & $14(30.4)$ & 656 (35.9) & \\
\hline Age $>50-60, \mathrm{n}(\%)$ & 9 (19.6) & $378(20.7)$ & \\
\hline Age $>60-70, \mathrm{n}(\%)$ & $12(26.1)$ & $266(14.6)$ & \\
\hline Age $>70, \mathrm{n}(\%)$ & $11(23.9)$ & $526(28.8)$ & 0.19 \\
\hline Male sex, n (\%) & $27(58.7)$ & 1067 (58.4) & 1.00 \\
\hline Diabetes, n (\%) & $8(17.4)$ & $190(10.4)$ & 0.20 \\
\hline Prior MI, n (\%) & 5 (10.9) & $118(6.5)$ & 0.37 \\
\hline COPD, n (\%) & $8(17.4)$ & $244(13.4)$ & 0.57 \\
\hline Hypertension, n (\%) & $13(28.3)$ & $318(17.4)$ & 0.088 \\
\hline Any malignancy, n (\%) & $8(17.4)$ & $242(13.3)$ & 0.55 \\
\hline Rheumatic disease, $\mathrm{n}(\%)$ & NA & NA & 1.00 \\
\hline
\end{tabular}

Abbreviations: COVID-19, Corona virus disease 2019; COPD, chronic obstructive pulmonary disease; MI, myocardial infarction; NA, not available (according to Statistics Denmark legislation, cells with number of patients below 3 cannot be reported).

Table 2. Propensity of ibuprofen treatment for COVID-19 patients. 


\begin{tabular}{|c|c|c|c|c|}
\hline Variable & Units & OR & $95 \% \mathrm{CI}$ & P-value \\
\hline \multirow[t]{4}{*}{ Age group } & $30-50$ & Ref & & \\
\hline & $>50-60$ & 0.97 & [0.41-2.29] & 0.94 \\
\hline & $>60-70$ & 1.54 & [0.66-3.59] & 0.32 \\
\hline & $>70-80$ & 0.56 & {$[0.22-1.45]$} & 0.23 \\
\hline \multirow[t]{2}{*}{ Sex } & Female & Ref & & \\
\hline & Male & 0.94 & {$[0.51-1.73]$} & 0.85 \\
\hline \multirow[t]{2}{*}{ Diabetes } & No & Ref & & \\
\hline & Yes & 1.56 & {$[0.67-3.65]$} & 0.30 \\
\hline \multirow[t]{2}{*}{ Prior MI } & No & Ref & & \\
\hline & Yes & 1.49 & {$[0.54-4.15]$} & 0.44 \\
\hline \multirow[t]{2}{*}{ COPD } & No & Ref & & \\
\hline & Yes & 1.32 & {$[0.60-2.92]$} & 0.49 \\
\hline \multirow[t]{2}{*}{ Hypertension } & No & Ref & & \\
\hline & Yes & 1.82 & {$[0.85-3.91]$} & 0.12 \\
\hline \multirow[t]{2}{*}{ Any malignancy } & No & Ref & & \\
\hline & Yes & 1.35 & [0.59-3.05] & 0.48 \\
\hline \multirow[t]{2}{*}{ Rheumatic disease } & No & Ref & & \\
\hline & Yes & 0.86 & {$[0.20-3.70]$} & 0.84 \\
\hline
\end{tabular}

Abbreviations: COVID-19, Corona virus disease 2019; OR, odds ratio; 95\% CI, confidence intervals; COPD, chronic obstructive pulmonary disease; MI, myocardial infarction.

Table 3. Results from logistic regression analyses to associate the risk of severe COVID-19 infection, death or intensive care unit admission with ibuprofen exposure, with no ibuprofen exposure as reference. 


\begin{tabular}{lccc}
\hline Model & OR & 95\% CI & P-value \\
\hline Crude & 1.77 & {$[0.91-3.47]$} & 0.093 \\
\hline Age-sex adjusted & 1.74 & {$[0.83-3.64]$} & 0.14 \\
\hline Fully adjusted & 1.57 & {$[0.72-3.38]$} & 0.25 \\
\hline
\end{tabular}

Abbreviations: COVID-19, Corona virus disease 2019; OR, odds ratio; 95\% CI, confidence intervals. The fully adjusted model included age (in categories: 30-50, >50-60, >60-70, >70 years), sex, diabetes, prior myocardial infarction, chronic obstructive pulmonary disease, hypertension, any malignancy and rheumatic disease

Figures

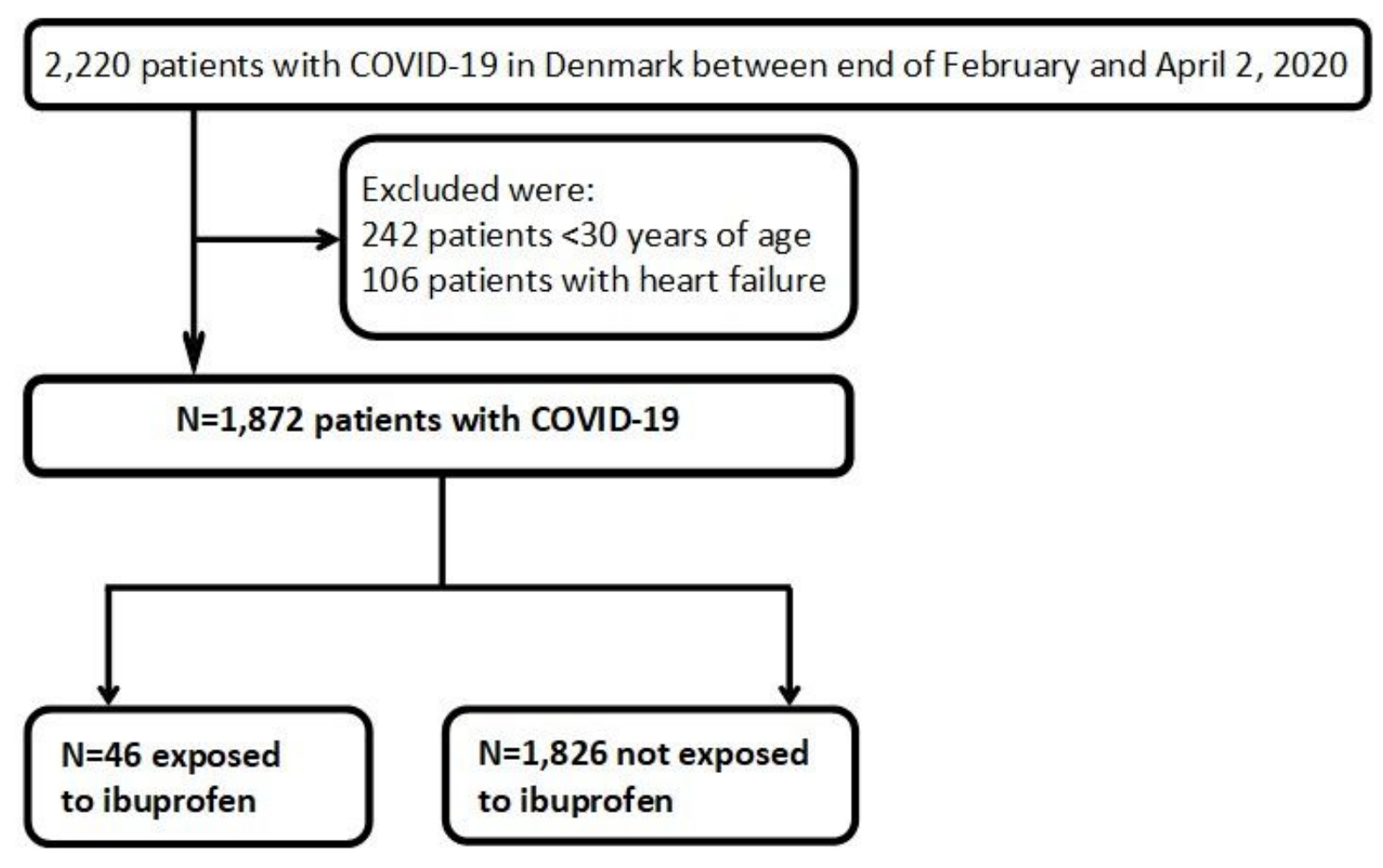

Figure 1 
Patient selection.

Page 13/13 